\title{
Location! The unanswered question in right ventricular pacing
}

\author{
Vineet Kumar, $M D^{a}$ \\ a Division of Cardiovascular Diseases, University of Alabama at Birmingham, Birmingham, AL
}

Received Dec 8, 2014; accepted Dec 8, 2014

doi: $10.1007 /$ s12350-014-0049-4

\section{See related article, pp. 903-911}

Location, like in real estate, is the most important question in electrophysiology as it determines the acute and long-term outcomes in patients undergoing ablation as well as placement of pacing or high voltage leads. Right ventricular (RV) apex is the most commonly selected location for placement of RV pacing leads. This preference reflects the ease, stability, and excellent pacing thresholds usually achieved at this location. However, human and experimental data have suggested potential deleterious effects of RV pacing on cardiac function, and thus, there has been an interest in alternate RV pacing sites. Singh et al in this issue of the journal present their study comparing right ventricular outflow tract (RVOT) and RV apical pacing using equilibrium radionuclide angiography (ERNA). To better understand and review the findings of their study or any trial of alternate site RV pacing, we must first answer the following questions.

\section{WHY RV APICAL PACING IS DELETERIOUS TO CARDIAC FUNCTION?}

RV apical pacing leads to iatrogenic left bundle branch block (LBBB) resulting in altered left ventricular activation pattern. To appreciate why this is deleterious to left ventricle (LV) performance, we should contrast it with normal LV endocardial activation. In normal hearts with intact conduction system, LV endocardial activation

Reprint requests: Vineet Kumar, MD, Division of Cardiovascular Diseases, University of Alabama at Birmingham, 930 Faculty Office Towers, 510 20th Street South, Birmingham, AL, 35294-0006; vkumar@uab.edu

J Nucl Cardiol 2015;22:912-5.

$1071-3581 / \$ 34.00$

Copyright (C) 2014 American Society of Nuclear Cardiology. occurs 0-15 ms after the onset of QRS almost simultaneously in two areas: the mid septum and the anterobasal wall. From here, it spreads briskly with total endocardial activation time of $30-50 \mathrm{~ms}$, which results in synchronous mechanical contraction of all areas in the LV. ${ }^{1}$ In pacing-induced $\mathrm{LBBB}$, the activation has to spread from right to left across the septum, which is slow, resulting in earliest $\mathrm{LV}$ endocardial activation around $50 \mathrm{~ms}$ after the onset of QRS and hence interventricular dyssynchrony. From here, the activation spreads across the rest of the LV with total endocardial activation time around $80-90 \mathrm{~ms}$. This time can be exaggerated in patients with underlying cardiomyopathy or previous infarction as reflected by markedly longer paced QRS duration in these patients resulting in significant intraventricular dyssynchrony. The inter- and intraventricular dyssynchrony from RV pacing results in acute decrease in cardiac pump function as shown by a decrease in the maximum dp/dt, mean arterial pressure, stroke volume, and stroke work and an increase in isovolumetric contraction and relaxation times in both animal and human studies. ${ }^{2-4}$ The pressure volume relationship of the LV shifts rightward and it operates at a higher volume. Dyssynchrony in activation and subsequent contraction of the LV leads to heterogeneity of stretch and loading conditions in pacing-induced or native LBBB. This can cause regional changes in myocardial perfusion as seen by perfusion defects and wall motion abnormalities in many of these patients with angiographically normal coronary arteries. ${ }^{5-7}$ These acute changes in $\mathrm{LV}$ performance when present over a long duration can lead to asymmetric hypertrophy, neurohormonal activation, and LV remodeling, all of which might contribute to increased incidence of heart failure, atrial fibrillation, and death observed in these patients. ${ }^{8-11}$

\section{WHY EVERYONE WITH RV APICAL PACING DOES NOT DEVELOP HEART FAILURE?}

Magnitude of the adverse effect of RV apical pacing in an individual depends on the interplay of several 
factors. These include (1) amount/percentage of RV pacing; (2) duration of pacing; and (3) paced QRS duration which in turn is determined by the underlying substrate. ${ }^{12,13}$ The last one is perhaps the most important. Patients with underlying cardiomyopathy or ischemic heart disease have longer paced QRS duration than patients without structural heart disease, suggesting poor cell to cell electrical coupling. Hence, the intraventricular dyssynchrony is exaggerated in these groups of patients, and they predictably have higher heart failure event rates with RV apical pacing. Majority of pacemaker implants are done in elderly population ${ }^{14}$ with expected lifetime burden of RV apical pacing being small; moreover, most of the patients receiving a pacemaker have normal LV structure and function resulting in a relatively narrow paced QRS. These groups of patients are able to tolerate RV apical pacing reasonably well. This is the reason why the incidence of heart failure linked to pacing is low in clinical trials, and it has been difficult to show significant differences in clinical outcomes. On the other hand, RV apical pacing in patients with depressed LV function and paced QRS duration greater than $160 \mathrm{~ms}$ is associated with more than $50 \%$ probability of heart failure over a 2-year period signifying the importance of underlying substrate which is paced in addition to the site of pacing. ${ }^{12,15}$

\section{WHAT ARE THE ALTERNATIVE RIGHT VENTRICULAR PACING STRATEGIES?}

What would be an ideal RV pacing site? An ideal site would induce minimum dyssynchrony by engaging the conduction system early, would be easily accessible trans-venously with the currently available tools for lead implantation, and would have stable pacing thresholds and lead position over long duration of follow-up. Hisian (pacing the His Bundle) and Para-hisian (pacing the myocardium around the His bundle) pacing although restores the normal electrical activation pattern, it is technically difficult and has problems with higher pacing thresholds. ${ }^{16,17}$ RVOT pacing has been studied and compared with RVA pacing in multiple trials with most of them favoring non-apical pacing sites. A metaanalysis of 14 randomized controlled trials with more than 700 patients showed higher left ventricular ejection fraction (LVEF) with non-apical pacing site. The difference in LVEF between apical and non-apical sites was most prominent in trials with greater than 12 months of follow-up and depressed baseline LVEF. ${ }^{18-20}$ Lack of uniform benefit in all trials can be attributed to selection of wrong site in the RVOT, short duration of follow-up, and smaller number of patients with normal underlying substrate. It is important to keep in mind that RVOT is a long tubular structure with many possible locations for pacing. Anterior free wall of the RVOT is easily accessible for lead placement but is anatomically distant from conduction system, pacing at this location, usually results in wide QRS, and may not represent the ideal site. Septal and posteroseptal RVOTs are relatively closer to the conduction system and therefore are better pacing sites. When the lead is implanted intentionally in the posteroseptal RVOT, the QRS duration is narrower consistent with more physiological and synchronous activation of the ventricles. ${ }^{21}$

From the discussion in the previous paragraphs, it can be understood why it would be very hard to show clinical benefit from alternate RV site pacing if we take patients with relatively normal LVEF and follow them for a relatively short period of time. This is the main reason why most pacing trials have had difficulty in showing clinical benefit of alternate site pacing. In addition, the lead position in most of the trials has not been optimized to ensure the narrowest possible QRS duration and minimum dyssynchrony which is the eventual aim.

In the current issue of the journal, Singh et al present their study looking at left ventricular function and mechanical dyssynchrony in patients with RVOT versus RV apical pacing using ERNA over a 12-month follow-up period. The strengths of the study are the use of ERNA as a method to asses LV function, which is a highly accurate and reproducible technique compared to echocardiography which has been used in most of the previous trials and can be subjective. They enrolled adequate number of patients requiring significant amount of RV pacing. They were able to show a significant decline in LVEF with RV apical pacing and maintenance of LV function with RVOT pacing. The decrease in LVEF was small, and therefore, no significant difference was seen between the two groups. The authors did not intentionally try to place the lead in posterior/posteroseptal RVOT but the paced QRS duration was significantly shorter from RVOT compared to RV apex which is reassuring. The study for the first time also shows the use of ERNA in assessing dyssynchrony between these two groups. Although no significant difference was found, there are several reasons for this observation. This was a relatively healthy population with normal left ventricular substrate as none of the patient's had coronary artery disease or depressed LVEF, so intraventricular dyssynchrony was small to start with as compared to patients with coronary artery disease or cardiomyopathy. There was no attempt to selectively place the lead at a location closer to the conduction system and minimize the duration of paced QRS and hence dyssynchrony. Same reasons may also explain why no significant difference in LV function was seen between the true groups at the end of study 
period. The duration of follow-up has to be much longer in a population with normal underlying substrate to show the deleterious effect of RV apical pacing. There were no clinical data in terms of hospital admissions, heart failure events, and mortality. Despite of these shortcomings of the trial, the use of ERNA to assess dyssynchrony in this population is a novel idea. Echocardiographic assessment of dyssynchrony as the authors point out has several limitations including reproducibility, standardization, and lack of agreement on which parameters to use. Ability to accurately and reproducibly quantify dyssynchrony using ERNA might help to predict which patients are at high risk for developing pacing-induced LV dysfunction. This highrisk group can then be targeted for intervention with either BiVentricular or alternate site RV pacing.

\section{CONCLUSION AND FUTURE DIRECTIONS}

We now know from human and animal studies that $\mathrm{RV}$ pacing is deleterious to cardiac function, and every effort should be made to minimize it. Strategies to minimize RV pacing include use of longer or dynamic $\mathrm{AV}$ delays in pacemakers, mode switch algorithms, and biventricular pacing in selected population. For patients who do require significant amount of pacing, decisions regarding lead placement have to be individualized. It seems RV apical pacing is well tolerated for short duration in individuals with normal left ventricular function and structure. Therefore, it might not be unreasonable to do this in an elderly male with normal LV function compared to someone who is anticipated to live several decades and needs significant amount of pacing or someone who has structurally abnormal LV. To conclusively show the difference between non-apical versus RV apical pacing, trial design will have to keep the following factors in consideration: location of the lead in non-apical position guided by anatomy and paced QRS duration, objective assessment of dyssynchrony, underlying left ventricular substrate, and amount of pacing which will be anticipated in these patients. These factors will determine the follow-up period which is necessary to show a significant difference. In conclusion, RV apical pacing is deleterious but the magnitude of this effect varies according to patient-specific factors. Assessment of dyssynchrony using a reproducible and objective method might help us in identifying the population at risk which may benefit from alternate pacing strategies.

\section{Disclosure}

I declare no conflict of interest.

\section{References}

1. Cassidy DM, Vassallo JA, Marchlinski FE, Buxton AE, Untereker WJ, Josephson ME. Endocardial mapping in humans in sinus rhythm with normal left ventricles: Activation patterns and characteristics of electrograms. Circulation 1984;70:37-42.

2. Nahlawi M, Waligora M, Spies SM, Bonow RO, Kadish AH, Goldberger JJ. Left ventricular function during and after right ventricular pacing. J Am Coll Cardiol 2004;44:1883-8.

3. Prinzen FW, Peschar M. Relation between the pacing induced sequence of activation and left ventricular pump function in animals. Pacing Clin Electrophys 2002;25:484-98.

4. Leclercq C, Gras D, Le Helloco A, Nicol L, Mabo P, Daubert C. Hemodynamic importance of preserving the normal sequence of ventricular activation in permanent cardiac pacing. Am Heart $\mathbf{J}$ 1995;129:1133-41.

5. Prinzen FW, Augustijn CH, Arts T, Allessie MA, Reneman RS. Redistribution of myocardial fiber strain and blood flow by asynchronous activation. Am J Phys 1990;259:H300-8.

6. Nowak B, Sinha AM, Schaefer WM, Koch KC, Kaiser HJ, Hanrath $\mathrm{P}$, et al. Cardiac resynchronization therapy homogenizes myocardial glucose metabolism and perfusion in dilated cardiomyopathy and left bundle branch block. J Am Coll Cardiol 2003;41:1523-8.

7. Kumar V, Venkataraman R, Aljaroudi W, Osorio J, Heo J, Iskandrian $\mathrm{AE}$, et al. Implications of left bundle branch block in patient treatment. Am J Cardiol 2013;111:291-300.

8. Lee MA, Dae MW, Langberg JJ, Griffin JC, Chin MC, Finkbeiner WE, et al. Effects of long-term right ventricular apical pacing on left ventricular perfusion, innervation, function and histology. J Am Coll Cardiol 1994;24:225-32.

9. Tse HF, Lau CP. Long-term effect of right ventricular pacing on myocardial perfusion and function. $\mathrm{J}$ Am Coll Cardiol 1997;29:744-9.

10. Sweeney MO, Hellkamp AS, Ellenbogen KA, Greenspon AJ, Freedman RA, Lee KL, et al. Adverse effect of ventricular pacing on heart failure and atrial fibrillation among patients with normal baseline QRS duration in a clinical trial of pacemaker therapy for sinus node dysfunction. Circulation 2003;107:2932-7.

11. Wilkoff BL, Cook JR, Epstein AE, Greene L, Hallstrom AP, Hsia $\mathrm{H}$, et al. Dual-chamber pacing or ventricular backup pacing in patients with an implantable defibrillator: The Dual Chamber and VVI Implantable Defibrillator (DAVID) Trial. J Am Med Assoc 2002;288:3115-23.

12. Sweeney MO, Hellkamp AS. Heart failure during cardiac pacing. Circulation 2006;113:2082-8.

13. Hayes JJ, Sharma AD, Love JC, Herre JM, Leonen AO, Kudenchuk PJ. Abnormal conduction increases risk of adverse outcomes from right ventricular pacing. J Am Coll Cardiol 2006;48:1628-33.

14. Mond HG, Irwin M, Morillo C, Ector H. The world survey of cardiac pacing and cardioverter defibrillators: Calendar year 2001. Pacing Clin Electrophys 2004;27:955-64.

15. Shukla HH, Hellkamp AS, James EA, Flaker GC, Lee KL, Sweeney MO, et al. Heart failure hospitalization is more common in pacemaker patients with sinus node dysfunction and a prolonged paced QRS duration. Heart Rhythm 2005;2:245-51.

16. Almendral Garrote J. His-bundle pacing: Great concept, difficult in practice. Rev Esp Cardiol 2006;59:534-6.

17. Kronborg MB, Mortensen PT, Poulsen SH, Gerdes JC, Jensen HK, Nielsen JC. His or para-His pacing preserves left ventricular function in atrioventricular block: A double-blind, randomized, crossover study. Europace 2014;16:1189-96.

18. Shimony A, Eisenberg MJ, Filion KB, Amit G. Beneficial effects of right ventricular non-apical vs. apical pacing: A systematic 
review and meta-analysis of randomized-controlled trials. Europace 2012;14:81-91.

19. Weizong W, Zhongsu W, Yujiao Z, Mei G, Jiangrong W, Yong Z, et al. Effects of right ventricular nonapical pacing on cardiac function: A meta-analysis of randomized controlled trials. Pacing Clin Electrophys 2013;36:1032-51.

20. Molina L, Sutton R, Gandoy W, Reyes N, Lara S, Limon F, et al. Medium-term effects of septal and apical pacing in pacemaker- dependent patients: A double-blind prospective randomized study. Pacing Clin Electrophys 2014;37:207-14.

21. Hillock RJ, Stevenson IH, Mond HG. The right ventricular outflow tract: A comparative study of septal, anterior wall, and free wall pacing. Pacing Clin Electrophys 2007;30:942-7. 